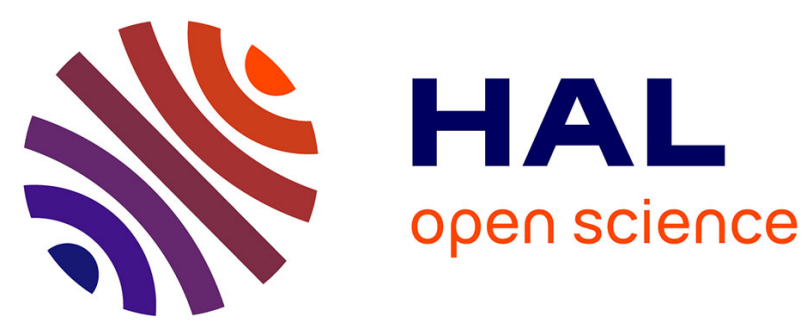

\title{
Universality and origin of ultrashort intrinsic negative dielectric permittivity
}

Sergey Prosandeev, Charles Paillard, B. Xu, L. Bellaiche

\section{To cite this version:}

Sergey Prosandeev, Charles Paillard, B. Xu, L. Bellaiche. Universality and origin of ultrashort intrinsic negative dielectric permittivity. Physical Review B: Condensed Matter and Materials Physics (19982015), 2020, 101, 10.1103/PhysRevB.101.024111 . hal-02482767

\section{HAL Id: hal-02482767 https://hal.science/hal-02482767}

Submitted on 18 Feb 2020

HAL is a multi-disciplinary open access archive for the deposit and dissemination of scientific research documents, whether they are published or not. The documents may come from teaching and research institutions in France or abroad, or from public or private research centers.
L'archive ouverte pluridisciplinaire HAL, est destinée au dépôt et à la diffusion de documents scientifiques de niveau recherche, publiés ou non, émanant des établissements d'enseignement et de recherche français ou étrangers, des laboratoires publics ou privés. 


\title{
Universality and origin of ultrashort intrinsic negative dielectric permittivity
}

\author{
Sergey Prosandeev, ${ }^{1,2}$ Charles Paillard $\odot,{ }^{1,3}$ B. Xu, ${ }^{4}$ and L. Bellaiche ${ }^{1}$ \\ ${ }^{1}$ Physics Department and Institute for Nanoscience and Engineering, University of Arkansas, Fayetteville, Arkansas 72701, USA \\ ${ }^{2}$ Institute of Physics and Physics Department of Southern Federal University, Rostov-na-Donu 344090, Russia \\ ${ }^{3}$ Laboratoire Structures, Propriétés et Modélisation des Solides, CentraleSupélec, UMR CNRS 8580, Université Paris-Saclay, \\ 91190 Gif-sur-Yvette, France \\ ${ }^{4}$ School of Physical Science and Technology, Soochow University, Suzhou 215006, China
}

(Received 26 October 2019; revised manuscript received 18 December 2019; published 24 January 2020)

\begin{abstract}
By recasting the definition of the dielectric constants in terms of currents, as well as using atomistic simulations and analytical derivations, we show that the dielectric permittivity can be negative at an ultrashort timescale, under perfect screening conditions and for very different materials and switching mechanisms, in line with recent experiments and modelings. In particular, we found that this effect can be due to a previously overlooked phenomenon of postswitching polarization oscillations. We also derived practical analytical formulas that can be experimentally checked.
\end{abstract}

DOI: 10.1103/PhysRevB.101.024111

\section{INTRODUCTION}

It was long thought, due to basic thermodynamic laws, that capacitance (which is proportional to the dielectric constant) ought to be positive (see what is probably the first discussion of this point in Ref. [1]). Recently, it was shown experimentally and by modeling polarization switching with the help of a two-well model $[2,3]$ that the "transient" capacitance during polarization switching in a ferroelectric could momentarily become negative due to negative curvature of the energy of the two-well model near the maximum between the wells. Reference [4] extended this explanation proposing that passing the negative curvature portion generates a strong depolarizing field. It is interesting that this conclusion sounds similar to the discovery that negative capacitance can exist even in statics, in a ferroelectric having dead layers $[5,6]$ or in heterostructures of ferroelectrics with dielectrics [7-11], due to a depolarizing field. Moreover, Ref. [12] extended the homogeneousswitching approach to the local $180^{\circ}$ domain's switching model in a system with some distribution of the parameters of the two-well systems over the domains. The transient negative capacitance in lead zirconium titanate (PZT) was then shown to originate from reverse domain nucleation and growth. The authors claimed that the capacitance becomes positive again after domain coalescence.

Here, we decided to perform calculations of the evolution of the transient negative dielectric permittivity (TNDP) during polarization switching in ferroelectrics by using a large-scale molecular-dynamics (MD) approach altogether with firstprinciples-based Hamiltonians describing interatomic interactions. We demonstrate that this effect can be an internal (material) phenomenon (that is, it does not have to be due to external resistors, capacitors, or inductions) and exists (i) irrespective of the switching mechanism (i.e., homogeneous and different types of inhomogeneous ones) in different ferroelectric materials, and (ii) can be traced back to the existence of specific nonequilibrium currents. The structure of the paper is as follows. In Sec. II, we provide definitions of quantities used in our study. In Sec. III, we discuss properties of the materials selected for calculations. In Sec. IV, we briefly describe the method of the calculation employed. In Sec. V we discuss the results of our calculations. In Sec. VI, we suggest how to verify our predictions experimentally. Finally, Sec. VII formulates our conclusions.

\section{DEFINITIONS VALID FOR NONEQUILIBRIUM}

Let us start by recalling that the thermodynamic properties and response functions of ferroelectrics depend on the Hamiltonian $\mathcal{H}=\mathcal{H}_{0}-\mathbf{E}_{0} \sum_{i} \mathbf{p}_{i}$ describing the energy terms at the microscopic (atomic) level. Here $\mathbf{E}_{0}$ is a homogeneous field acting on (and thus interacting with) each local dipole, as commonly done for theoretical descriptions of polarization switching in ferroelectrics [13], and $\mathbf{p}_{i}$ is an electric dipole at site $i$. The Hamiltonian we employ describes interactions between electric dipoles and between these dipoles and other degrees of freedom such as strain, magnetic moments, oxygen octahedra tilting, etc. We consider here an infinite periodic system having ideal screening conditions, because of periodic boundary conditions. In the molecular-dynamics (MD) method, each individual dipole $\boldsymbol{p}_{i}$ evolves according to classical (Newtonian) equations of motion [14-16],

$$
m_{i} \ddot{\boldsymbol{p}}_{i}=-\frac{\partial \mathcal{H}_{0}}{\partial \boldsymbol{p}_{i}}+\boldsymbol{E}_{0}
$$

with $m_{i}$ being a mass associated with a dipole $\boldsymbol{p}_{i}$ (an analog of a "displacement" in Newtonian mechanics), and $\ddot{\boldsymbol{p}}_{i}$ denotes the second time derivative of $\boldsymbol{p}_{i}(t)$ ("accelerations"). "Velocities", $\dot{\boldsymbol{p}}$, in this approach, can be found from the general kinetic equations and initial conditions. "Viscosity" appears naturally, because of the potential barriers in the dynamical path of the system [13]. 
The term $\boldsymbol{e}_{i}=\left(\frac{\partial \mathcal{H}_{0}}{\partial \boldsymbol{p}_{i}}\right)$ can be viewed as an on-site electric field. Let us now introduce a vector of electric field, $\boldsymbol{E}$, as the average, over the sites " $i$," of these on-site fields: $\boldsymbol{E}=\left\langle\boldsymbol{e}_{i}\right\rangle$. Note that, at equilibrium, the instant value of the electric field in the ferroelectric coincides with the homogeneous field $\boldsymbol{E}_{0}$ (Ref. [7]), that is, $\boldsymbol{E}=\boldsymbol{E}_{0}$, because, at equilibrium, the average of the "generalized force" [the right-hand side of Eq. (1); see Ref. [14]] over $i$ must vanish. However, these two fields, $\boldsymbol{E}$ and $\boldsymbol{E}_{0}$, can be different away from equilibrium, because in the transient state the average "generalized force" does not have to be zero. The measure of this difference is thus the nonequilibrium field $\boldsymbol{E}_{\text {neq }}=\boldsymbol{E}-\boldsymbol{E}_{0}$. This is one of the most important ideas of the present study. Note that $\boldsymbol{E}=\boldsymbol{E}_{0}+\boldsymbol{E}_{\text {neq }}$ is the field defining the instant voltage drop on the ferroelectric, namely $V_{F}=t_{F} E$ (Ref. [3]), where $t_{F}$ is the thickness of the ferroelectric capacitor and $E$ is the instant electric field across the capacitor.

The instant value of the electric displacement vector can now be defined as $\boldsymbol{D}=\boldsymbol{P}+\varepsilon_{0} \boldsymbol{E}$, where $\varepsilon_{0}$ is the vacuum permittivity and $\boldsymbol{P}$ is the instant (not necessarily equilibrium) value of the average volume density of the dipole moments of the system.

Further on, one may define the instant value of the relative dielectric permittivity in a nonequilibrium state as

$$
\varepsilon=\frac{d D}{\varepsilon_{0} d E}
$$

For the sake of simplicity, we consider the changes of the electric displacement and electric field as projections on the direction of field $\boldsymbol{E}_{0}$ applied to the sample to switch polarization. Interestingly, Eq. (2) depends not only on the change of the user-controlled field $\boldsymbol{E}_{0}$, but also on the change of the instant nonequilibrium fields $\boldsymbol{E}$ and $\boldsymbol{E}_{\text {neq. }}$. In particular, during out-of-equilibrium switching of the polarization, the instant electric field can spontaneously change due to the destabilization or/and excitation of optical phonons or relaxators, even under a constant electric field $\boldsymbol{E}_{0}$, until the system reaches its new equilibrium position.

Importantly, $\varepsilon$ can be recast in terms of the current induced by the electric displacement, $\dot{D}$, and of the current associated with the electric field, $\varepsilon_{0} \dot{E}$ :

$$
\varepsilon=\frac{\dot{D}}{\varepsilon_{0} \dot{E}} .
$$

This formula reduces the measurement of the momentary dielectric constant to the measurement of momentary currents, as a function of time. We reckon from Eq. (3) that TNDP is achieved when one of the two following criteria is met:

$$
\begin{aligned}
& \dot{E}<0, \dot{D}>0 \text {; or equivalently } \dot{P}>-\varepsilon_{0} \dot{E} \text { and } \dot{E}<0, \\
& \dot{E}>0, \dot{D}<0 \text {; or equivalently } \dot{P}<-\varepsilon_{0} \dot{E} \text { and } \dot{E}>0 .
\end{aligned}
$$

Specifically, if the current associated with the electric field, $\dot{E}$, is opposite to the current associated with the electric displacement field, $\dot{D}$, the instant relative dielectric permittivity becomes negative. Equivalently, if the current associated with the polarization, $\dot{P}$, is opposite and larger in magnitude than the current associated with the electric field, the instant relative dielectric permittivity becomes negative.
Note that in terms of measurable quantities, $\dot{D}=\dot{q}_{\text {free }} / A$ and $\dot{E}=\dot{V}_{F} / t_{F}$, where $\dot{q}_{\text {free }}$ is the free-charge current, $\dot{V}_{F}$ is the current of the voltage on the ferroelectric, and $A$ is the area of the capacitor. For example, Ref. [4] formulates the same conditions as (4) and (5) in the following way: $\dot{P}>\dot{Q}_{\text {free }}>0$ and $\dot{P}<\dot{Q}_{\text {free }}<0$, where $Q_{\text {free }}=q_{\text {free }} / A$.

\section{MATERIALS SELECTED FOR CALCULATIONS}

We selected two materials: lead magnesium niobate (PMN) and bismuth ferrite (BFO). PMN is a ferroelectric relaxor $[17,18]$. It shows an anomalous birefringence temperature dependence below the so-called Burns temperature [19]. Further cooling down results in freezing phenomena, which nevertheless do not result in a macroscopic polarization [18]. However, PMN can also adopt a polar phase when poled at low temperature under an electric bias field [18,20,21]. An unconventional polarization switching dynamics has recently been predicted in PMN (Ref. [20]) in which first a so-called infinite cluster (a region of correlated dipoles spreading throughout the whole supercell) melts and breaks into smaller isolated polar clusters. The latter then rotate and switch their local polarization before merging together to form a new infinite cluster with a switched polarization. Interestingly, this picture is close qualitatively to the picture described in Ref. [12] in their modeling of domain type switching in PZT, but, instead of domains, we found percolation clusters.

Moreover, BFO is a well-known multiferroic material with numerous complex and intertwined degrees of freedom [22]. BFO in its supertetragonal phase (called T-BFO here) can show either homogeneous switching (HS) or nucleationlimited switching [13] (NLS), depending on the magnitude of the external electric field [23].

\section{METHODS}

In 1994, first-principles-based calculations showed that ferroelectrics can be accurately modeled by an effective Hamiltonian $\mathcal{H}$ (see Ref. [24]) that employs only a limited number of degrees of freedoms, such as microscopic electric dipoles $\boldsymbol{p}_{i}$ and local and global strains.

For PMN: We conducted simulations on an $18 \times 18 \times 18$ supercell by using the effective Hamiltonian developed in Ref. [25]. This effective Hamiltonian reproduces several specific temperatures inherent to relaxor systems and proposes that relaxor behavior originates from the competition between random fields (arising from the random distribution of the $\mathrm{Mg}$ and $\mathrm{Nb}$ ions in the supercell, as done here with the help of a random number generator) and peculiar antiferroelectric interactions. Local and average polarization can then be defined by proper summing and division by the volume of the local dipole moments.

Here, our setup is the same as in Ref. [20]. In other words, we first pole PMN along the [111] pseudocubic direction using a $5 \mathrm{MV} / \mathrm{cm}$ external electric field. Then we relax this poled state during 40000 Monte-Carlo (MC) sweeps. We then employ the molecular-dynamics method in which we study the polarization reversal by applying a bias field of $4 \mathrm{MV} / \mathrm{cm}$ along [1 $1 \overline{1} \overline{1}]$. All calculations ran at a temperature of $10 \mathrm{~K}$. 
PMN

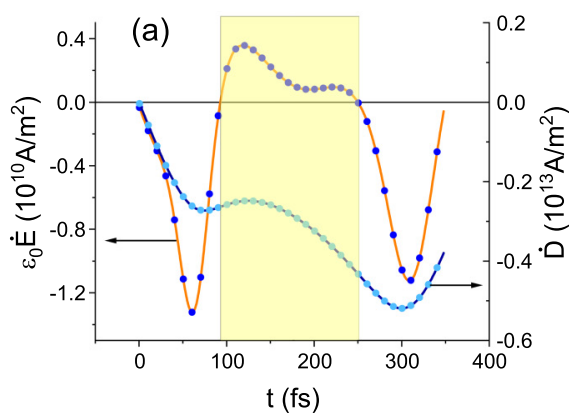

(d)

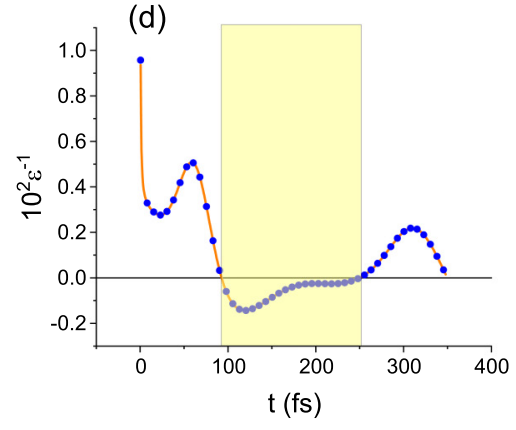

T-BFO NLS

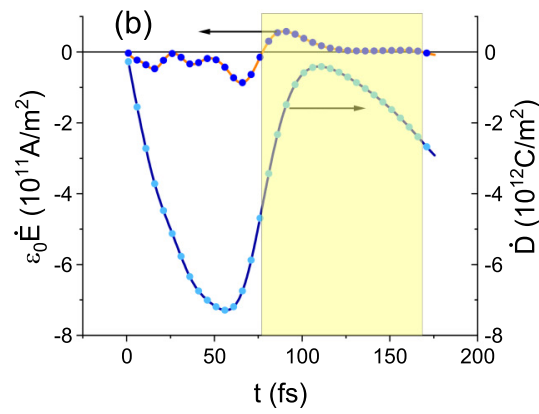

(e)

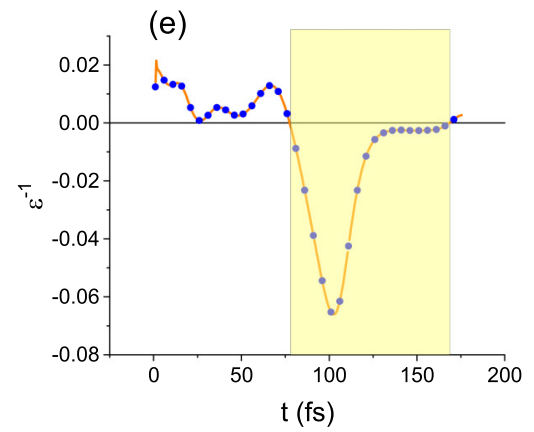

T-BFO HS
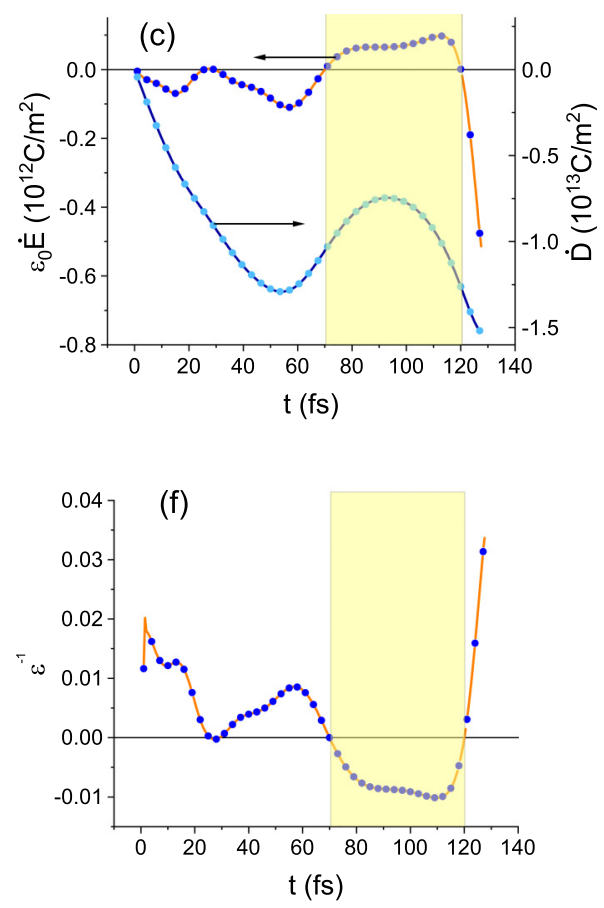

FIG. 1. Properties related to TNDP. The regions of TNDP are marked by yellow shaded areas. (a)-(c) Time evolution of different displacement currents in PMN (a), T-BFO with NLS mechanism (b), and T-BFO with HS mechanism (c); (d)-(f) Time evolution of the inverse dielectric permittivity in PMN (d), T-BFO with NLS mechanism, (e) and T-BFO with HS mechanism (f).

For BFO: The effective Hamiltonian developed in Refs. [23,26,27] is used to investigate $\mathrm{BiFeO}_{3}$ in its supertetragonal phase. A $48 \times 48 \times 6$ supercell is used, and the homogeneous strain components $\eta_{H, 1}$ and $\eta_{H, 2}$ are clamped to impose a $-6 \%$ compressive strain with respect to their relaxed bulk values in the $R 3 c$ phase; concurrently, $\eta_{H, 6}$ is fixed to zero. From the ferroelectric state relaxed with Monte Carlo simulations, we conduct MD simulations with a time step of $0.5 \mathrm{fs}$, at a temperature of $10 \mathrm{~K}$, and we apply an electric field opposite to the initial polarization with magnitude either (i) $25 \mathrm{MV} / \mathrm{cm}$ to get NLS, or (ii) $40 \mathrm{MV} / \mathrm{cm}$ to induce $\mathrm{HS}$. Note that, due to Landauer's paradox, the measured coercive field is often much smaller than the theoretical prediction. For instance, a factor of $\sim 25$ has been found in the $R 3 c$ phase of BFO (see Ref. [23]). Such rescaling would make the 25 and $40 \mathrm{MV} / \mathrm{cm}$ used in our simulations smaller than the experimental coercive field of about 2.2-4.3 MV/cm reported for BFO films in Ref. [28].

\section{RESULTS OF CALCULATIONS}

Comparing in Figs. 1(a)-1(c) the current associated with the electric displacement field, $\dot{D}$, with the current induced by the instant electric field, $\dot{E}$, one observes that they have opposite signs in the yellow shaded intervals for PMN and TBFO for NLS, and T-BFO for HS, respectively. Figures 1(d)1(f) present the inverse of the dielectric permittivities calculated by formula (3) for the same materials and types of switching. We see that these quantities are negative in the same intervals, and we thus witness the emergence of TNDP caused by polarization switching, in two different ferroelectric compounds (poled PMN and T-BFO) and for three different switching mechanisms (in particular NLS versus HS in BFO). At the same time, the criterion exposed in Eqs. (4) and (5), namely that if the current associated with the electric field, $\dot{E}$, is opposite to the current associated with the electric displacement field, $\dot{D}$, the instant relative dielectric permittivity becomes negative, is evidently satisfied in the shaded areas, as seen from Figs. 1(a)-1(c).

We also performed a run of the computation of the polarization switching in PMN that is longer than the one shown Fig. 1. The results of such a longer run are presented in Fig. 2.

One can clearly see a periodic oscillation of the polarization after $\simeq 320 \mathrm{fs}$, that is, after its switching-with this oscillation decreasing in magnitude as time increases. The time period of such oscillations can be extracted from Fig. 2(a) and equals $345 \mathrm{fs}$, which therefore corresponds to a natural frequency of $f=2.9 \mathrm{THz}$. This latter frequency is consistent with the phonon frequency of about $2.7 \mathrm{THz}$ reported in Ref. [29] and assigned there to be an $A$ transverse optical mode. It is thus legitimate to assume that the polarization explores the harmonic potential around its minimum in energy but with this exploration away from the minimum being reduced as time evolves, in order to interpret the oscillation of the polarization after switching and its further decrease in magnitude in time.

Strikingly, Fig. 2(b) reveals that these phonon-induced periodic oscillations of the polarization give rise to TNDP at the time-points for which the polarization is basically maximum or minimum during these oscillations. These short-time intervals of TNDP are due to viscosity that introduces a slight 
(a)

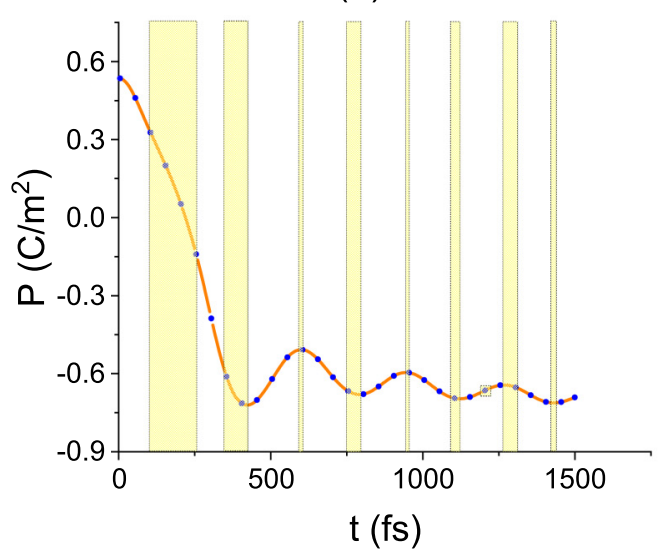

(b)

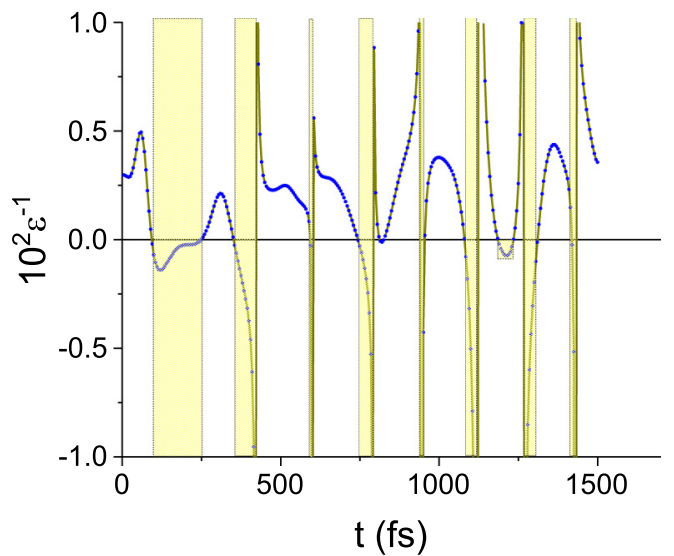

FIG. 2. Time evolution of some physical quantities in PMN. Panel (a) shows the polarization in a long MD run, while panel (b) depicts the inverse of the dielectric response in that long run.

phase shift between the harmonic vibrations of $\dot{D}$ and $\varepsilon_{0} \dot{E}$; see Fig. 3. Consequently, the inverse of the internal dielectric permittivity, $\varepsilon^{-1}=\varepsilon_{0} \dot{E} / \dot{D}$, has thin intervals of TNDP in which the conditions (4) and (5) are satisfied.

To explain the phase shift and emergence of additional peaks in the $E(t)$ dependence, as compared to the $D(t)$ dependence, let us concentrate on Fig. 3 close to 500 fs. One can indeed see a single peak in $\dot{D}$ at $t=505 \mathrm{fs}$ as well as a corresponding peak in $\dot{E}$ at that precise time. They correspond to positive $\varepsilon$ as this follows from Eq. (3), at which both $\dot{D}$ and $\dot{E}$ are positive. In addition, there is also a plateau in $\dot{E}$ at $t=385 \mathrm{fs}$. This plateau lies in the left yellow shaded bar of a time region exhibiting TNDP, and for which $\dot{D}$ and $\dot{E}$ are now of opposite sign - with $\dot{D}$ now being negative while $\dot{E}$ is positive and nearly insensitive to time (hence the plateau).

Note also that we obtained (not shown here) similar polarization oscillations in both BFO NLS and HS switching mechanisms, with these oscillations also resulting in many

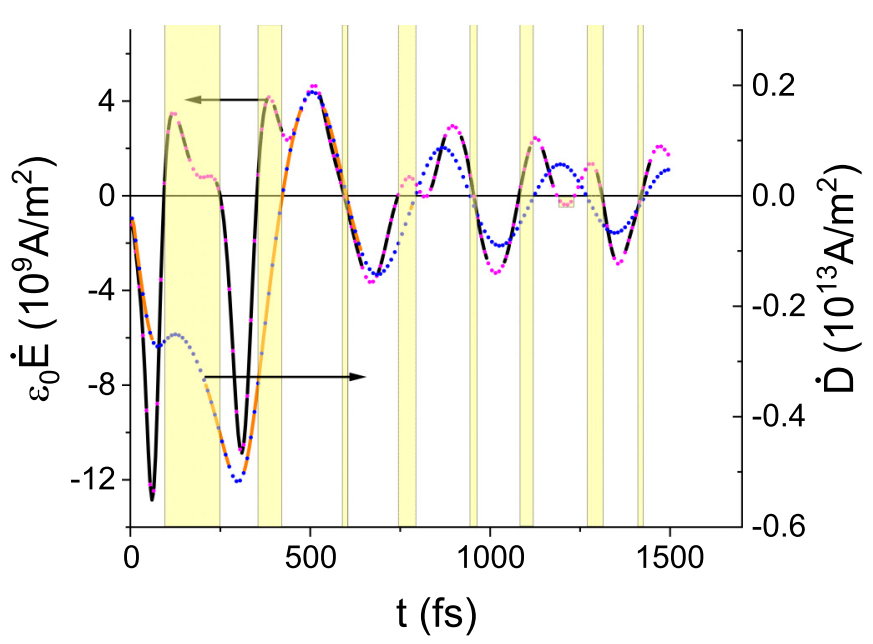

FIG. 3. Time evolution of the currents $\dot{D}$ and $\varepsilon_{0} \dot{E}$ in PMN shows that local maxima and minima of the polar oscillations (after $320 \mathrm{fs}$ ) exhibit slight delays between $\dot{D}$ and $\varepsilon_{0} \dot{E}$, leaving a window where they have opposite signs. short-time TNDP intervals- therefore demonstrating the generality of such an effect.

Note that the understanding of these oscillations can be easily achieved in the frame of the harmonic oscillator model in the underdamped regime, which is inherent, e.g., to ringing in RLC electric circuits [30]. In the present case, the role of induction is played by inertia of the dipoles during the switching, which has not been taken into account in previous modelings.

\section{HOW TO VERIFY TNDP EXPERIMENTALLY?}

Let us now consider a specific electric circuit whose schematization is shown in Fig. 4. Application of Ohm's law to this circuit gives

$$
V_{0}=I R+V_{F}
$$

where $V_{0}$ is the battery voltage, $I$ is the electric current, $R$ is the resistance, and $V_{F}$ is the potential drop on the ferroelectric capacitor (we assume here that the contacts at the ferroelectric are perfect in order to avoid consideration of stripe domains). Note that Eq. (6) is common in many studies [2-4].

Now, let us plug the formula $V_{F}=t_{F} E$ into Eq. (6) (see Ref. [3]), then differentiate this equation with respect to time, divide the result by $I$, and take into account that

$$
I=A \dot{D}
$$

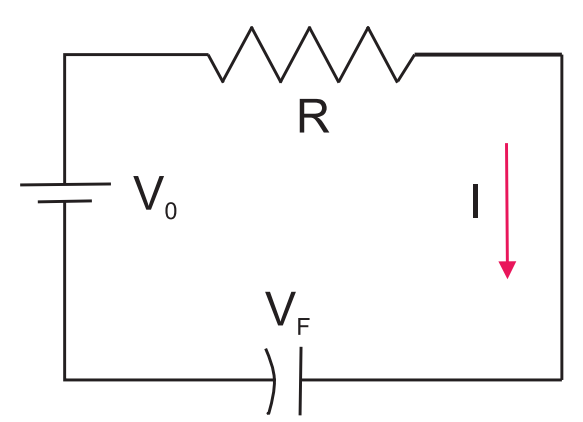

FIG. 4. A sketch of an experimental electric circuit. 
where $A$ is the area of the ferroelectric capacitor. We then obtain

$$
\frac{\dot{V}_{0}}{I}=\frac{\dot{I} R}{I}+\frac{t_{F} \dot{E}}{A \dot{D}}
$$

One can recall from Sec. II that

$$
\frac{\varepsilon_{0} \dot{E}}{\dot{D}}=\varepsilon^{-1} \text {. }
$$

Then Eq. (8) takes the form

$$
\frac{\dot{V}_{0}}{I}=\frac{\dot{I} R}{I}+\frac{t_{F} \varepsilon^{-1}}{\varepsilon_{0} A} .
$$

Now we can solve Eq. (10) with respect to $\varepsilon^{-1}$ :

$$
\varepsilon^{-1}=\frac{\varepsilon_{0} A \dot{V}_{0}}{t_{F} I}-\frac{A R \varepsilon_{0} \dot{I}}{t_{F} I}
$$

The first term of this equation is finite if only the voltage on the battery changes in time. The second term indicates that, when $V_{0}$ is constant with time, TNDP can occur as a result of positive $\frac{\dot{I}}{\bar{I}}$.

It is interesting to compare the last finding with experiment. For example, in Ref. [2] electric current in the interval of TNDP has either a positive first derivative of $I$ with respect to time and positive values of $I$, or a negative first derivative of $I$ and negative values of $I$. This is in line with the negative values of the dielectric permittivity given by our Eq. (11).

Note that, by employing Eq. (6), one can also express $\varepsilon^{-1}$ via $V_{F}$ and its time derivative:

$$
\varepsilon^{-1}=\frac{\varepsilon_{0} A R}{t_{F}\left(V_{0}-V_{F}\right)} \dot{V}_{F} .
$$

This is a very interesting result: One can keep $\dot{V}_{0}$ constant or not constant, but $\varepsilon^{-1}$ depends only on $\dot{V}_{F}$ and $V_{0}$ but not on $\dot{V}_{0}$.

Let us now describe one of the possible reasons for TNDP, when $\frac{i}{I}$ is positive. For that, we now employ the simple model of Ref. [2], which substitutes the electric field (voltage) on the ferroelectric by a Landau expansion:

$$
E=\alpha D+\beta D^{3} .
$$

Then Eq. (6) can be rewritten in the form

$$
V_{0}=R I+t_{F}\left(\alpha D+\beta D^{3}\right) .
$$

Taking the first time derivative of this equation and solving the result with respect to $\dot{I}$, after also taking into account Eq. (7), yields

$$
\dot{I}=-\frac{t_{F}\left(\alpha+3 \beta D^{2}\right) \dot{D}-\dot{V}_{0}}{R} .
$$

Plugging this result into Eq. (11) gives

$$
\varepsilon^{-1}=\varepsilon_{0}\left(\alpha+3 \beta D^{2}\right) .
$$

Note that the result, again, does not depend on $\dot{V}_{0}$. So, the dielectric permittivity is negative when $D^{2}<\frac{-\alpha}{3 \beta}$. In this interval, the curvature of the energy, $F=\frac{1}{2} \alpha D^{2}+\frac{1}{4} \beta D^{4}-E_{0} D$, with respect to the electric displacement is negative.

Thus, within the simple one-dimensional model considering a homogeneous polarization switching, one can obtain that (i) the inequality $\frac{I}{I}>0$ is possible, for example if $D^{2}<\frac{-\alpha}{3 \beta}$, which corresponds to the totally unstable transient states; (ii) one of the possible reasons for TNDP is dynamically overcoming an interval where the curvature of energy versus electric displacement is negative. The latter statement was published earlier based on a different derivation and is in accord with experiments [2,3]. However, one should be careful with this idea because the system in this transient state is totally unstable, and polar phonons are also unstable. From this point of view, this transient state, achieved during the switching, cannot exist in statics, but it will probably be possible to stabilize it in the future with the help of a resonator.

Getting the inverse dielectric permittivity from a measurement of the electric current and voltage on the ferroelectric is not the only benefit of such a measurement. One can have more. Specifically, by measuring the voltage drop on the ferroelectric [or by expressing it from Eq. (6)], one can scan the energy relief. Indeed, the electric free energy can be obtained from the integral

$$
\Delta U=\int^{t} E \dot{D} d t
$$

Let us rewrite this integral in terms of experimental observables:

$$
\begin{aligned}
\Delta U & =\frac{1}{A t_{F}} \int^{t} V_{F} I(t) d t=\frac{1}{A R t_{F}} \int^{t} V_{F}\left(V_{0}-V_{F}\right) d t \\
& =\frac{1}{A t_{F}} \int^{t} I\left(V_{0}-I R\right) d t .
\end{aligned}
$$

\section{CONCLUSION}

We derived analytically the most general conditions for TNDP, which are consistent with previously derived conditions in Ref. [4]. These conditions impose some inequalities involving the currents of the nonequilibrium instant electric field we introduced and of instant electric displacement (or polarization). Our first-principles-based calculations performed for two different systems with different types of polarization switching confirm the existence of TNDP in finite-time intervals as an internal property of materials at nonequilibrium. We found out that one of the possible reasons for TNDP is postswitching polarization oscillations (that are reminiscent of ringing in RLC electric circuits [30]). We have also developed a simple analytical model and derived practical formulas to measure TNDP to be checked further in experiment.

\section{ACKNOWLEDGMENTS}

S.P. acknowledges ONR Grant No. N00014-17-1-2818, and the support from Grant RMES No. 3.1649.2017/4.6. C.P. acknowledges the support of ARO Grant No. W911NF161-0227. B.X. and L.B. acknowledge the DARPA Grant No. HR0011-15-2-0038 (MATRIX program). B.X. also acknowledges the startup fund from Soochow University, and the support from Priority Academic Program Development (PAPD) of Jiangsu Higher Education Institutions. We also acknowledge the computational support from Arkansas High Performance Computer Center at the University of Arkansas. 
[1] R. Landauer, Collect. Phenom. 2, 167 (1976).

[2] A. I. Khan, K. Chatterjee, B. Wang, S. Drapcho, L. You, C. Serrao, S. R. Bakaul, R. Ramesh, and S. Salahuddin, Nat. Mater. 14, 182 (2015).

[3] M. Hoffmann, F. P. G. Fengler, M. Herzig, T. Mittmann, B. Max, U. Schroeder, R. Negrea, P. Lucian, S. Slesazeck, and T. Mikolajick, Nature (London) 565, 464 (2019).

[4] S.-C. Chang, U. E. Avci, D. E. Nikonov, S. Manipatruni, and I. A. Young, Phys. Rev. Appl. 9, 014010 (2018).

[5] A. M. Bratkovsky and A. P. Levanyuk, Phys. Rev. B 63, 132103 (2001).

[6] A. M. Bratkovsky and A. P. Levanyuk, Appl. Phys. Lett. 89, 253108 (2006).

[7] P. Zubko, J. C. Wojdeł, M. Hadjimichael, S. Fernandez-Pena, A. Sené, I. Lukyanchuk, J.-M. Triscone, and J. Íñiguez, Nature (London) 534, 524 (2016).

[8] A. K. Yadav, K. X. Nguyen, Z. Hong, P. García-Fernández, P. Aguado-Puente, C. T. Nelson, S. Das, B. Prasad, D. Kwon, S. Cheema, A. I. Khan, C. Hu, J. Íñiguez, J. Junquera, L.-Q. Chen, D. A. Muller, R. Ramesh, and S. Salahuddin, Nature (London) 565, 468 (2019).

[9] I. Lukyanchuk, Y. Tikhonov, A. Sené, A. Razumnaya, and V. M. Vinokur, Commun. Phys. 2, 22 (2019).

[10] J. Íñiguez, P. Zubko, I. Luk'yanchuk, and A. Cano, Nat. Rev. Mater. 4, 243 (2019).

[11] H. W. Park, J. Roh, Y. B. Lee, and C. S. Hwang, Adv. Mater. 5, 1805266 (2019).

[12] M. Hoffmann, A. I. Khan, C. Serrao, Z. Lu, S. Salahuddin, M. Pešić, S. Slesazeck, U. Schroeder, and T. Mikolajick, J. Appl. Phys. 123, 184101 (2018).

[13] A. K. Tagantsev, I. Stolichnov, N. Setter, J. S. Cross, and M. Tsukada, Phys. Rev. B 66, 214109 (2002).
[14] D. Rapaport, The Art of Molecular Dynamics Simulation, (Cambridge University Press, Cambridge, 2001).

[15] V. Boddu, F. Endres, and P. Steinmann, Sci. Rep. 7, 806 (2017).

[16] P. S. Chen and C. W. Liu, Mater. Res. Express 6, 095045 (2019).

[17] G. A. Smolenskii, V. A. Bokov, V. A. Isupov, N. N. Krainik, R. E. Pasynkov, and A. I. Sokolov, Ferroelectrics and Related Materials (Gordon and Breach, New York, 1981).

[18] L. E. Cross, Ferroelectrics 151, 305 (1994).

[19] G. Burns and F. H. Dacol, Phys. Rev. B 28, 2527 (1983).

[20] S. Prosandeev, B. Xu, and L. Bellaiche, Phys. Rev. B 98, 024105 (2018).

[21] X. Zhao, W. Qu, X. Tan, A. A. Bokov, and Z.-G. Ye, Phys. Rev. B 75, 104106 (2007).

[22] G. Catalan and J. F. Scott, Adv. Mater. 21, 2463 (2009).

[23] B. Xu, V. Garcia, S. Fusil, M. Bibes, and L. Bellaiche, Phys. Rev. B 95, 104104 (2017).

[24] W. Zhong, D. Vanderbilt, and K. M. Rabe, Phys. Rev. Lett. 73, 1861 (1994).

[25] A. Al-Barakaty, S. Prosandeev, D. Wang, B. Dkhil, and L. Bellaiche, Phys. Rev. B 91, 214117 (2015).

[26] I. A. Kornev, L. Bellaiche, P. E. Janolin, B. Dkhil, and E. Suard, Phys. Rev. Lett. 97, 157601 (2006).

[27] D. Albrecht, S. Lisenkov, W. Ren, D. Rahmedov, I. A. Kornev, and L. Bellaiche, Phys. Rev. B 81, 140401(R) (2010).

[28] H. Yamada, V. Garcia, S. Fusil, S. Boyn, M. Marinova, A. Gloter, S. Xavier, J. Grollier, E. Jacquet, C. Carrétéro, C. Deranlot, M. Bibes, and A. Barthélémy, ACS Nano 7, 5385 (2013).

[29] A. Al-Zein, J. Hlinka, J. Rouquette, and B. Hehlen, Phys. Rev. Lett. 105, 017601 (2010).

[30] D. L. Eggleston, Basic Electronics for Scientists and Engineers (Cambridge University Press, Cambridge, 2011). 